\title{
Genetic Polymorphism of GABRG2 rs2 I I037 is Associated with Drug Response and Adverse Drug Reactions to Valproic Acid in Chinese Southern Children with Epilepsy
}

\author{
Jieluan Lu' \\ Hanbing $\mathrm{Xia}^{2}$ \\ Wenzhou $\mathrm{Li}^{2}$ \\ Xianhuan Shen' \\ Huijuan Guo ${ }^{2}$ \\ Jianping Zhang' \\ Xiaomei Fan (iD ${ }^{2}$ \\ 'Department of Clinical Pharmacology, \\ College of Pharmacy, Jinan University, \\ Guangzhou, 510632, People's Republic of \\ China; ${ }^{2}$ Department of Pharmacy, Baoan \\ Women's and Children's Hospital, Jinan \\ University, Shenzhen, 518102, People's \\ Republic of China
}

Correspondence: Jianping Zhang Department of Clinical Pharmacology,

College of Pharmacy, Jinan University, 60I

W. Huangpu Avenue, Guangzhou, 51 0632,

People's Republic of China

Tel +862085220261

Email tzhangjp@jnu.edu.cn

Xiaomei Fan

Department of Pharmacy, Baoan

Women's and Children's Hospital, Jinan

University, 56\# Yulv Road, Baoan District,

Shenzhen, 518102, People's Republic of

China

Tel +8675527863999

Email xmfane@I63.com
Background: Valproic acid (VPA) is recommended as a first-line treatment for children with epilepsy. GABRG2 polymorphism is found to be associated with epilepsy susceptibility and therapeutic response of anti-seizure medications (ASM); however, the role of GABRG2 in VPA treatment still remains unknown.

Objective: The purpose of this study was to explore the association of GABRG2 gene polymorphism with the drug response and adverse drug reactions (ADRs) related to VPA.

Methods: A retrospective study including 96 Chinese children with epilepsy treated by VPA was carried out. The ADRs were collected during VPA therapy and GABRG2 rs211037 in enrolled patients was genotyped using Sequenom MassArray system. A network pharmacological analysis involved protein-protein interaction and enrichment analysis was constructed to investigate the potential targets and pathways of GABRG2 on VPA-related ADRs. Results: Among 96 patients, 41 individuals were defined as seizure together with 49 patients with seizure-free and 6 patients unclassified. Carriers of homozygote GABRG2 rs 211037 CC genotype exhibited seizure-free to VPA $(P=0.042)$, whereas those with CT genotype showed seizure. Furthermore, CC genotype had predisposition to digestive ADRs $(P=0.037)$ but was a protective factor for VPA-associated weight gain $(P=0.013)$. Ten key genes related to digestive ADRs and weight gain induced by VPA were identified by network pharmacological analysis and mainly involved in "GABAergic synaptic signaling", "GABA receptor signaling", and "taste transduction" pathways/processes through enrichment analysis.

Conclusion: This study revealed that $G A B R G 2$ variation exerted a predictable role in the efficacy and safety of VPA treatment for Chinese children with epilepsy.

Keywords: children with epilepsy, GABRG2, gene polymorphism, valproic acid, therapeutic response, adverse drug reactions

\section{Introduction}

Epilepsy, characterized by persistent epileptic seizures, has a prevalence of 7.60 per 1000 individuals worldwide and affects around 70 million people in all age groups. ${ }^{1}$ Epilepsy is one of the most common neurologic disorders in children with the highest incidence in the first year after birth, and the comorbidities are also common in children with epilepsy. ${ }^{2}$ Anti-seizure medications (ASM) are the first choice for the treatment of epilepsy in both children and adults, represented by valproic acid (VPA) with the broadest effects against epilepsy compared with other ASM. 
VPA, metabolized mainly by glucuronidation, betaoxidation, and cytochrome P450-mediated oxidation, is effective in all types of epileptic seizures and syndromes. ${ }^{3}$ However, over $30 \%$ of children with epilepsy showed recurrent seizure to VPA. ${ }^{4-6}$ Non-responsiveness /ineffectiveness and adverse drug reactions (ADRs) have become the most common prevalent reasons for epileptic patients to discontinue VPA treatment. Digestive adverse reactions (dADRs) including appetite changes, vomiting or nausea, diarrhea, and dysphagia, and weight gain are usually observed in epileptic patients treated by VPA. ${ }^{7-9}$ Evidences have indicated that genetic abnormalities may lead to non-responsiveness or ineffectiveness to VPA for specific individuals via regulation of channel activities, drug pharmacokinetics, and neuronal excitability. ${ }^{10}$ VPA can intensify synthesis and release of gammaaminobutyric (GABA), resulting in an increase in the transmission of GABAergic via specific brain regions. ${ }^{11,12}$ Interestingly, GABA as an inhibitory neurotransmitter implicates the alteration of mesolimbic systems. VPA also regulates the activity of GABA receptors including $\mathrm{GABA}_{\mathrm{A}}$ and $\mathrm{GABA}_{\mathrm{B}}$, extending the elimination of inhibitory ability based on $\mathrm{GABA}_{\mathrm{A}}$ receptor activation. $^{13,14}$ GABRG2, a primary mutation in the $\mathrm{GABA}_{\mathrm{A}}$ receptor $\gamma 2$ subunit, is a significant locus associated with a majority of epilepsy and drug response among idiopathic generalized epilepsies, febrile seizures, and childhood absence epilepsy. ${ }^{15-18}$ The polymorphism of GABRG2 rs211037 increases the susceptibility of idiopathic generalized epilepsy and may regulate the response to ASM in the Macedonian population and Egyptian children. ${ }^{19,20}$ However, no relationship was found between GABRG2 rs211037 and the susceptibility of Juvenile myoclonic epilepsy. ${ }^{21}$ GABRG2 gene variation may influence the predisposition to epilepsy in juvenile myoclonic epilepsy regardless of its phenotype. ${ }^{22}$ The TT homozygote of $G A B R G 2$ rs211037 was reported to be a predictive genetic marker of febrile seizures and generalized recurrent seizures and to predispose patients treated by ASM to recurrent seizures. ${ }^{23,24}$ No significant linkage between GABRG2 rs211037 polymorphism and drug response to ASM was found in Indians. ${ }^{25}$ However, the role of GABRG2 in ADRs related to ASM remains unknown.

In this study, a retrospective study in Chinese southern children with epilepsy was performed to evaluate whether the polymorphism of GABRG2 rs211037 is associated with VPA response and ADRs. Target pathway/function network of GABRG2 in ADRs was explored through network pharmacological analysis to predict the underlying mechanisms.

\section{Materials and Methods}

\section{Participants and Data Collection}

A total of 96 children with epilepsy were gathered in Baoan Women's and Children's Hospital from October 2016 to January 2020. The epilepsy or epileptic syndrome were diagnosed on the bias of the latest ILAE Commission's classification standard. ${ }^{26}$ The children with epilepsy aged $<16$ years old who received VPA alone or in combination of the other ASM (LEV, OXC, TPM, and LTG) were included in this study. All the children were administered with VPA at least 12 months. Patients who were diagnosed as epilepsy within a month of birth were excluded. Those with a history of pseudoepileptic seizure, impaired hepatic and/or renal function, or the existence of any diseases which presented gastrointestinal symptoms similar to side effects induced by ASM were also excluded. The study was approved by the Baoan Women's and Children's Hospital Ethics Committee and conducted in accordance with the Declaration of Helsinki. Written informed consents were obtained from all patients' guardians. The clinical data of children with epilepsy were collected through the inpatients' medical records or a telephone interview with their parents. The information including gender, age of initial diagnosis, birth and family history of epilepsy, classification of epilepsy, ASM (only VPA or polytherapy), seizure frequency, and ADRs was collected.

\section{Drug Response and ADR Evaluation}

Seizure frequency was used in the classifications of drug response according to EpiPGX (https://www.epipgx.eu/) ${ }^{27}$ to evaluate the efficacy of VPA treatment. The individuals without epileptic seizure under ongoing therapy with VPA for at least 12 months were defined as seizure-free. Seizure was defined due to recurrent epileptic seizures in the course of VPA treatment with sufficient dosage, which was within the recommended dose range to obtain the therapeutic blood concentration $(50-100 \mu \mathrm{g} / \mathrm{mL}) .{ }^{28}$ The individuals who suffered ADRs when taking VPA were included in VPA induced-ADR group and further divided into four subgroups including neurogenic ADRs (nADRs) group, digestive ADRs (dADRs), weight-gain group, and cutaneous ADRs (cADRs) according to the clinical 
symptoms of ADRs. The group without ADRs was composed of patients who showed no ADRs related to VPA.

Causal assessment for ADRs developed following VPA administration was carried out using WHO-UMC methodology and the Naranjo Algorithm. All the ADRs were categorized as certain, probable/likely, possible, conditional/unclassified, and unassessable/unclassifiable. Only the ADRs in certain, probable/likely, and possible categories were included in this study.

\section{SNP Genotyping}

Genomic DNA samples were extracted from $1.5 \mathrm{~mL}$ of whole blood in all participants. The polymerase chain reaction was performed as described in detail in our previous study. ${ }^{29}$ Genotyping of GABRG2 rs211037 was carried out using iPLEX $^{\circledR}$ Gold Assay and Sequenom MassArray System (Agena Bioscience, San Diego, CA, United States). The MassArray Typer 4.0 software was used for data acquisition and analysis. The genotyping primers of GABRG2 $\mathrm{C}>\mathrm{T}$ rs211037 (forward primer sequence: ACGTTGGATGTACCATCTTGGCTTCT GGTGR and reverse primer sequence: ACGTTGGATGA GCTTCTGTCTGTCAGGTCGE) were specifically synthesized in our study.

\section{Analysis of Protein-Protein Interaction Network SNP Genotyping}

The GABRG2 was inputted to STRING database v11.01 (https://string-db.org/) to obtain protein-protein interaction (PPI) network. Only Homo sapiens proteins linked to GABRG2 were used for PPI analysis. The number of GABRG2-interacted proteins in the PPI network was restricted by setting an interaction score value with default medium confidence $(0.400)$ as cutoff criteria and the number of interactors no more than 20. The visible network figures were pictured using Cytoscape (v3.6.0) software (http://www.cytoscape.org/).

\section{Identification of GABRG2-Related Target Genes in VPA-Induced ADRs}

ADRs-related genes were obtained from DisGeNET (https://www.disgenet.org/) and GeneCards database (https://www.genecards.org/). The keywords such as "appetite change", "stomach upset" which referred to VPA-induced ADRs were used, and only Homo sapiens genes were considered in this study. Genes with a relevance score $\geq 1$ were selected in GeneCards database, and those with PubMed IDs $>0$ were included in DisGeNET database. ${ }^{29}$ Genes associated with VPA were obtained from GeneCards and DrugBank, and the overlapping genes were removed. All the ADR-related genes from the above two databases and VPA-associated genes were both intersected with the genes related to $G A B R G 2$ from PPI analysis, and $G A B R G 2$-associated target genes in VPA-induced ADRs were obtained.

\section{Pathway and Functional Enrichment Analysis}

The Kyoto Encyclopedia of Genes and Genomes (KEGG) and Gene Ontology (GO) analysis were performed using Metascape online tool (http://metascape.org/gp/index. $\underline{\mathrm{html}}$ ) in this study for exploring pathway enrichment and potential biological processes annotation of $G A B R G 2$ in VPA-induced ADRs. Only terms with a $P$-value less than 0.01 and enrichment factor $>1.5$ were considered significant.

\section{Statistical Analysis}

Independent-samples $t$-test was used to assess the differences of demographic characteristics between patients with and without VPA-induced ADRs. The data were expressed as mean \pm standard deviation (mean \pm SD). The allelic and genotypic frequencies for GABRG2 rs211037 were calculated by the SNPStats software (https://www.snpstats.net/). The chi-square test was used to evaluate the Hardy-Weinberg equilibrium (HWE). The differences of genotype frequencies for GABRG2 rs211037 between the seizure-free group and seizure group to VPA and between children with epilepsy with and without VPA-induced ADRs were analyzed by chi-square test or Fisher's exact test using SPSS V25.0. The OR and 95\% confidence interval $(\mathrm{CI})$ were calculated. $P<0.05$ was considered to be statistically significant.

\section{Results}

\section{Clinical Characteristics of Patients with Epilepsy}

Ninety-six children with epilepsy were involved in this study with 55 males and 41 females, who aged from 1 month to 16 years old with the average ages of diagnosis for $3.71 \pm 3.11$ years (Table 1). There were 47 individuals receiving VPA alone and 49 patients administered with VPA combined with other ASM including LEV (26.0\%), OXC (15.6\%), TPM $(5.2 \%)$, and LTG $(4.2 \%)$. More than half of patients with 
Table I Clinical Characteristics of Children with Epilepsy Receiving Valproic Acid

\begin{tabular}{|c|c|c|}
\hline Characteristics & Number (n) & Percentage (\%) \\
\hline \multicolumn{3}{|l|}{$\begin{array}{l}\text { Age of initial diagnosis } \\
\text { (years) }\end{array}$} \\
\hline$<2$ & 48 & 50.0 \\
\hline $2-16$ & 48 & 50.0 \\
\hline Mean \pm SD & $3.71 \pm 3.11$ & \\
\hline Male & 55 & 57.3 \\
\hline Female & 41 & 42.7 \\
\hline \multicolumn{3}{|l|}{ Drug treatment } \\
\hline VPA monotherapy & 47 & 49.0 \\
\hline Combination with other ASM & 49 & 51.0 \\
\hline Levetiracetam & 25 & 26.0 \\
\hline Oxcarbazepine & 15 & 15.6 \\
\hline Topiramate & 5 & 5.2 \\
\hline Lamotrigine & 4 & 4.2 \\
\hline \multicolumn{3}{|l|}{ Treatment response } \\
\hline Seizure & 41 & 42.7 \\
\hline Seizure-free & 49 & 51.0 \\
\hline Unclassified & 6 & 6.3 \\
\hline \multicolumn{3}{|l|}{ Adverse drug reactions } \\
\hline Digestive ADRs & 50 & $37.3^{\mathrm{a}}$ \\
\hline Appetite change & 13 & 9.7 \\
\hline Abdominal pain & 7 & 5.2 \\
\hline Stomach upset & II & 8.2 \\
\hline Nausea, vomiting, or both & 6 & 4.5 \\
\hline Diarrhea/Constipation & 13 & 9.7 \\
\hline Neurogenic ADRs & 38 & 28.4 \\
\hline Sleep problem & 8 & 6.0 \\
\hline Headache/ Dizziness & 5 & 3.7 \\
\hline Asthenia & 5 & 3.7 \\
\hline Irritability & 4 & 3.0 \\
\hline Somnolence & 6 & 4.5 \\
\hline Fatigue & 10 & 7.5 \\
\hline Weight gain & 28 & 20.9 \\
\hline Rash & 18 & 13.4 \\
\hline
\end{tabular}

${ }^{\text {a Notes: }}{ }^{\text {a }}$ The percentage of ADRs was calculated with 134 cases as the denominator.

epilepsy showed seizure-free after VPA administration. Idiopathic generalized epilepsy was the main type both in seizure-free group (73.5\%) and seizure group (53.7\%). The other six children were unclassified due to lack of clinical data. Children with epilepsy who showed seizure after VPA treatment tended to be younger than those with seizure-free to VPA treatment $(P=0.02)$. Gender of patients exhibited no effect on VPA efficacy (Table 2). The patients with VPA alone exhibited a higher percentage of seizure-free to VPA
Table 2 The Characteristics of Patients with Epilepsy Who Showed Seizure and Seizure-Free Treated by VPA

\begin{tabular}{|c|c|c|c|}
\hline & $\begin{array}{l}\text { Seizure } \\
(n=4 I)\end{array}$ & $\begin{array}{l}\text { Seizure-Free } \\
(n=49)\end{array}$ & $P$ value \\
\hline \multicolumn{4}{|l|}{$\begin{array}{l}\text { Age of initial } \\
\text { diagnosis (years) }\end{array}$} \\
\hline$<2$ & 17 (4I.5\%) & 29 (59.2\%) & 0.053 \\
\hline $2-16$ & 24 (58.5\%) & $20(40.8 \%)$ & \\
\hline Mean \pm SD & $3.13 \pm 2.34$ & $3.40 \pm 3.50$ & $0.021^{*}$ \\
\hline \multicolumn{4}{|l|}{ Gender } \\
\hline Male & 26 (63.4\%) & 29 (59.2\%) & 0.879 \\
\hline Female & 15 (36.6\%) & $20(40.8 \%)$ & \\
\hline \multicolumn{4}{|l|}{ Drug treatment } \\
\hline VPA monotherapy & 19 (34.5\%) & $36(65.5 \%)$ & $0.048^{*}$ \\
\hline Polytherapy & $22(62.9 \%)$ & $13(37.1 \%)$ & \\
\hline \multicolumn{4}{|l|}{$\begin{array}{l}\text { Blood concentration } \\
\text { of VPA }(\mu \mathrm{g} / \mathrm{mL})\end{array}$} \\
\hline Mean \pm SD & $61.6 \pm 20.3$ & $60.6 \pm 18.6$ & 0.830 \\
\hline \multicolumn{4}{|l|}{ Epilepsy types } \\
\hline Idiopathic generalized & $22(53.7 \%)$ & 36 (73.5\%) & \\
\hline Versus focal & 14 (34.1\%) & 7 (14.3\%) & \\
\hline Symptomatic & $3(7.3 \%)$ & 5 (10.2\%) & \\
\hline Status epilepsy & $2(4.9 \%)$ & I (2.0\%) & \\
\hline
\end{tabular}

Note: $* P<0.05$.

compared to those with polytherapy (65.5\% vs $37.1 \%$ ). There are no significant difference on VPA blood concentration in epileptic patients with or without seizure-free to VPA, which was measured using enzyme multiplied immunoassay technique.

A total of 134 ADRs of different systems or organs were observed in fifty children with epilepsy, indicating several children with epilepsy suffered from more than one side effect and were correspondingly included in several ADR groups. The different ADR symptoms per system or organ related to VPA were shown in Table 1. The percentages of "certain", "probable", and "possible" ADRs through causality assessment were $24.6 \%, 66.4 \%$, and $9 \%$, respectively. The main ADRs of patients were dADRs (37.3\%), nADRs (28.4\%), weight gain (20.9\%), and rash $(13.4 \%)$.

\section{The Association of GABRG2}

\section{Polymorphism with Drug Response to VPA in Children with Epilepsy}

The frequency distribution of GABRG2 rs211037 genotype in children with epilepsy receiving VPA was summarized 
Table 3 Comparison of GABRG2 Rs2 I 1037 Genotype Distribution in Children with Epilepsy with or without Response to VPA

\begin{tabular}{|c|c|c|c|c|c|}
\hline Genetic Model & Genotype & Seizure $(n=4 I)$ & Seizure-Free $(n=49)$ & $P$ value & OR $(95 \% \mathrm{Cl})$ \\
\hline Allele contrast & C vs $T$ & $\begin{array}{l}34(41.0 \%) / \\
48(59.0 \%)\end{array}$ & $\begin{array}{l}45(46.0 \%) / \\
53(54.0 \%)\end{array}$ & 0.549 & $\begin{array}{l}1.00 \\
0.84(0.46-1.5 I)\end{array}$ \\
\hline Codominant & CC vs CT vs TT & $\begin{array}{l}3(7.3 \%) / \\
28(68.3 \%) / \\
10(24.4 \%)\end{array}$ & $\begin{array}{l}\text { II (22.4\%) / } \\
23(47.0 \%) \text { / } \\
\text { I5 (30.6\%) }\end{array}$ & 0.059 & $\begin{array}{l}1.00 \\
4.46(1.11-17.93) \\
2.44(0.54-11.03)\end{array}$ \\
\hline Dominant & $\mathrm{CC}$ vs $\mathrm{CT}+\mathrm{TT}$ & $\begin{array}{l}3(7.3 \%) / \\
38(92.7 \%)\end{array}$ & $\begin{array}{l}\text { II (22.4\%) / } \\
38 \text { (77.5\%) }\end{array}$ & $0.042 *$ & $\begin{array}{l}1.00 \\
3.67(0.95-14.19)\end{array}$ \\
\hline Recessive & $\mathrm{CC}+\mathrm{CT}$ vs TT & $\begin{array}{l}31 \text { (75.6\%) / } \\
10(24.4 \%)\end{array}$ & $\begin{array}{l}34(69.4 \%) / \\
15(30.6 \%)\end{array}$ & 0.51 & $\begin{array}{l}1.00 \\
0.73(0.29-1.87)\end{array}$ \\
\hline Overdominant & $\mathrm{CC}+\mathrm{TT}$ vs CT & $\begin{array}{l}13(31.7 \%) / \\
28(68.3 \%)\end{array}$ & $\begin{array}{l}26(53.1 \%) / \\
23(46.9 \%)\end{array}$ & $0.040 *$ & $\begin{array}{l}1.00 \\
2.43(1.03-5.78)\end{array}$ \\
\hline Log-additive & CC vs TT & $\begin{array}{l}3(7.3 \%) / \\
10(24.4 \%)\end{array}$ & $\begin{array}{l}\text { II (22.4\%) / } \\
\text { I5 (30.6\%) }\end{array}$ & 0.51 & $\begin{array}{l}1.00 \\
1.24(0.65-2.36)\end{array}$ \\
\hline
\end{tabular}

Note: $* \mathrm{P}<0.05$

in Table 3. Frequency of $\mathrm{CC}$ genotype was higher in seizure than that in seizure-free $(22.4 \%$ and $7.3 \%$, respectively) through the dominant analysis. The individuals with seizure had a higher frequency in CT genotype than those with seizure-free to VPA $(68.3 \%$ and $46.9 \%$ respectively). No significant differences in the codominant, recessive, or log-additive models were observed between seizure group and seizure-free group. The results indicated that heterozygous CT genotype of GABRG2 rs211037 polymorphism tended to be a significant risk factor for seizure to VPA in children with epilepsy, but CC genotype exhibited seizure-free.

\section{The Association of GABRG2}

\section{Polymorphism with VPA-Induced dADRs and Weight-Gain in Children with \\ Epilepsy}

The association between GABRG2 rs211037 variants and VPA-induced ADRs was further investigated in our study. GABRG2 rs211037 polymorphism was found to be significantly associated with VPA-induced dADRs and weight gain, but had no linkage with nADRs and cADRs. The frequency distribution of $G A B R G 2$ rs211037 genotype in children with epilepsy with or without dADRs is shown in Table 4. C allele was more frequent in children with epilepsy with VPA-induced dADRs than those without dADRs $(51.0 \%$ vs $35.0 \%, P=0.028)$ as evidenced by the allele analysis. CC genotype had a significantly higher frequency in children with epilepsy with dADRs compared to those without dADRs. These results indicated that the allele $\mathrm{C}$ and homozygous $\mathrm{CC}$ genotype were risk factors for VPA-induced dADRs in children with epilepsy. Additionally, no significant difference in the frequency of $\mathrm{C}$ and $\mathrm{T}$ alleles was found among the patients with or without VPA-related weight gain $(P=0.0630)$ (Table 5). A significantly lower frequency of homozygous $\mathrm{CC}$ genotype was observed in children with VPA-induced weight gain compared to those without this ADRs $(3.6 \%$ vs $22.1 \%$ ), conversely for the genotypes with at least one $\mathrm{T}$ allele (CT or TT). These suggested that carriers with $\mathrm{CC}$ genotype might exhibit fewer predispositions to VPArelated weight gain.

\section{Network Pharmacological Analysis of GABRG2 in VPA-Induced dADRs and Weight Gain}

To explore the underlying mechanisms of GABRG2 in VPAinduced dADRs and weight gain, network pharmacological analysis was carried out in this study. A PPI network for GABRG2 comprising 20 nodes and 146 edges was generated (Figure 1A). Twenty genes were found to interact with GABRG2. A total of 10,282 genes associated with digestive system manifestations including "appetite change", "stomach upset", and "abdominal pain", and weight gain were obtained from DisGeNET and GeneCards. After intersecting with VPA-related genes from GeneCards and Drugbank, 924 genes were further intersected with 20 GABRG2-related 
Table 4 Comparison of GABRG2 Rs2 I I 037 Genotype Distribution in Children with Epilepsy with or without VPA-Induced dADRs

\begin{tabular}{|c|c|c|c|c|c|}
\hline $\begin{array}{l}\text { Genetic } \\
\text { Model }\end{array}$ & Genotype & $\begin{array}{l}\text { Children with Epilepsy } \\
\text { with dADRs }(n=35)\end{array}$ & $\begin{array}{l}\text { Children with Epilepsy } \\
\text { without dADRs }(n=6 I)\end{array}$ & $P$ value & OR $(95 \% \mathrm{CI})$ \\
\hline \multirow[t]{2}{*}{ Allele contrast } & \multirow[t]{2}{*}{ C vs T } & $36(51.0 \%) /$ & $43(35.0 \%) /$ & \multirow{2}{*}{$0.028^{*}$} & 1.00 \\
\hline & & 34 (49.0\%) & 79 (65.0\%) & & $1.95(1.07-3.60)$ \\
\hline \multirow[t]{3}{*}{ Codominant } & \multirow[t]{3}{*}{ CC vs CT vs TT } & $10(28.6 \%) /$ & $6(9.8 \%) /$ & \multirow{3}{*}{0.054} & 1.00 \\
\hline & & $16(45.7 \%) /$ & $31(50.8 \%) /$ & & I.38 (0.52-3.65) \\
\hline & & $9(25.7 \%)$ & $24(39.3 \%)$ & & $4.44(1.25-15.82)$ \\
\hline \multirow[t]{2}{*}{ Dominant } & \multirow[t]{2}{*}{$\mathrm{CT}+\mathrm{CC}$ vs.TT } & $26(74.3 \%) /$ & $37(60.7 \%) /$ & \multirow{2}{*}{0.17} & 1.00 \\
\hline & & $9(25.7 \%)$ & $24(39.3 \%)$ & & I.87 (0.75-4.68) \\
\hline \multirow[t]{2}{*}{ Recessive } & \multirow[t]{2}{*}{$\mathrm{CC}$ vs $\mathrm{CT}+\mathrm{TT}$} & $10(28.6 \%) /$ & $6(9.8 \%) /$ & \multirow{2}{*}{$0.037 *$} & 1.00 \\
\hline & & $25(71.4 \%)$ & $55(90.1 \%)$ & & $3.67(1.23-11.66)$ \\
\hline \multirow[t]{2}{*}{ Overdominant } & \multirow[t]{2}{*}{$\mathrm{CC}+\mathrm{TT}$ vs $\mathrm{CT}$} & $19(54.3 \%) /$ & $30(49.1 \%) /$ & \multirow{2}{*}{0.63} & 1.00 \\
\hline & & $16(45.7 \%)$ & 31 (50.8\%) & & $0.81(0.35-1.87)$ \\
\hline \multirow[t]{2}{*}{ Log-additive } & \multirow[t]{2}{*}{ CC vs TT } & $10(28.6 \%) /$ & $6(9.8 \%) /$ & \multirow{2}{*}{$0.027^{*}$} & 1.00 \\
\hline & & $9(25.7 \%)$ & 24 (39.3\%) & & $4.44(1.33-13.72)$ \\
\hline
\end{tabular}

Note: $* \mathrm{P}<0.05$.

Table 5 Comparison of GABRG2 Rs2 I I037 Genotype Distribution in Children with Epilepsy with or without VPA-Induced Weight Gain

\begin{tabular}{|c|c|c|c|c|c|}
\hline $\begin{array}{l}\text { Genetic } \\
\text { Model }\end{array}$ & Genotype & $\begin{array}{l}\text { Children with Epilepsy } \\
\text { with Weight Gain }(n=28)\end{array}$ & $\begin{array}{l}\text { Children with Epilepsy } \\
\text { without Weight Gain } \\
(n=68)\end{array}$ & $P$ value & OR (95\% Cl) \\
\hline \multirow[t]{2}{*}{ Allele contrast } & \multirow[t]{2}{*}{ C vs $T$} & $17(30.0 \%) /$ & $61(45.0 \%) /$ & \multirow{2}{*}{0.063} & 1.00 \\
\hline & & $39(70.0 \%)$ & 75 (55.0\%) & & $0.54(0.27-1.03)$ \\
\hline \multirow[t]{3}{*}{ Codominant } & \multirow[t]{3}{*}{$\mathrm{CC}$ vs $\mathrm{CT}$ vs TT } & I (3.6\%) / & $15(22.1 \%) /$ & \multirow{3}{*}{$0.046^{*}$} & 1.00 \\
\hline & & $15(53.5 \%) /$ & $31(45.6 \%) /$ & & $5.81(0.8 \mathrm{I}-65.95)$ \\
\hline & & $12(42.9 \%)$ & $22(32.4 \%)$ & & $7.64(1.29-83.48)$ \\
\hline \multirow[t]{2}{*}{ Dominant } & \multirow[t]{2}{*}{$\mathrm{CT}+\mathrm{CC}$ vs $\mathrm{TT}$} & $16(57.1 \%) /$ & $46(67.7 \%) /$ & \multirow{2}{*}{0.33} & 1.00 \\
\hline & & $12(42.9 \%)$ & $22(32.4 \%)$ & & $1.57(0.63-3.88)$ \\
\hline \multirow[t]{2}{*}{ Recessive } & \multirow[t]{2}{*}{$\mathrm{CC}$ vs.TT + CT } & I (3.6\%)/ & $15(22.1 \%) /$ & \multirow{2}{*}{$0.013^{*}$} & 1.00 \\
\hline & & 27 (96.4\%) & 53 (77.9\%) & & $7.64(1.29-83.48)$ \\
\hline \multirow[t]{2}{*}{ Overdominant } & \multirow[t]{2}{*}{$\mathrm{CC}+\mathrm{TT}$ vs $\mathrm{CT}$} & I3 (46.4\%) / & 37 (54.4\%) / & \multirow{2}{*}{0.48} & 1.00 \\
\hline & & $15(53.5 \%)$ & $31(45.6 \%)$ & & $0.73(0.30-1.76)$ \\
\hline \multirow[t]{2}{*}{ Log-additive } & \multirow[t]{2}{*}{ CC vs TT } & I (3.6\%) / & 15 (22.1\%) / & \multirow{2}{*}{0.06} & 1.00 \\
\hline & & $12(42.9 \%)$ & $22(32.4 \%)$ & & $1.88(0.95-3.70)$ \\
\hline
\end{tabular}

Note: $* P<0.05$. 
A

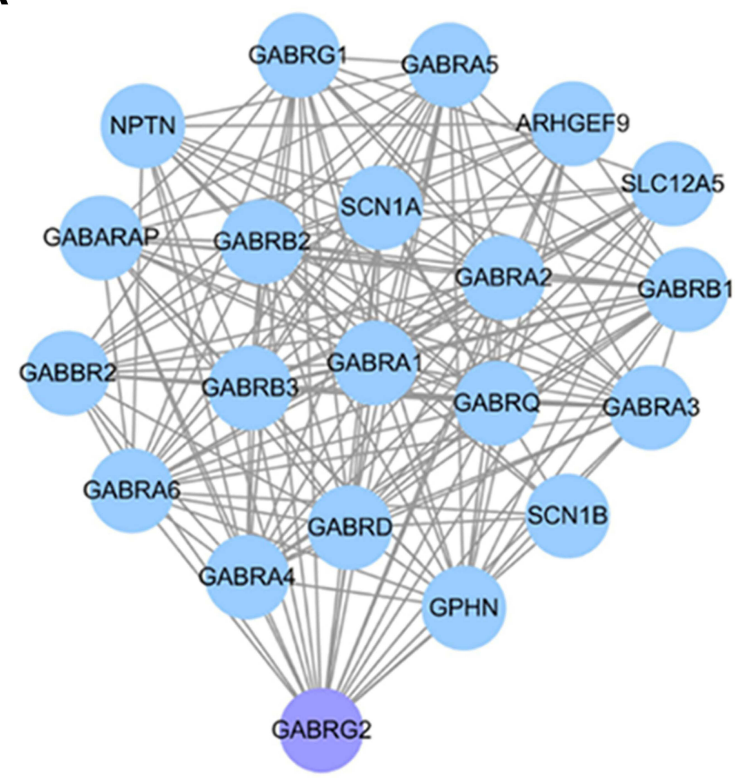

B

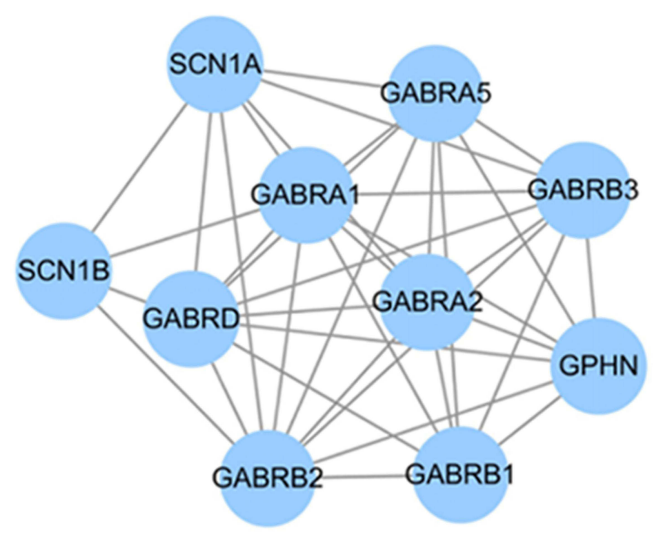

Figure I Protein-protein interaction (PPI) network analysis. (A) PPI network analysis of GABRG2 consisted of 20 nodes and I46 edges. (B) The PPI network for ten common target genes of GABRG2 in VPA-induced ADRs. Each target gene is represented by nodes and the association between two nodes is represented by lines.

genes from PPI network analysis. Ten common genes including $S C N 1 B, S C N 1 A, G A B R B 1, G A B R A 1, G A B R A 5$, GABRB3, GPHN, GABRA2, GABRD, and GABRB2 were identified as the key target genes of GABRG2 in VPAinduced dADRs and weight gain (Figure 1B).

To further investigate the biological functions of ten target genes, GO enrichment analysis was performed through Metascape. As shown in Figure 2A, the top 5 biological processes (BP) which 10 target genes were mainly enriched in were "gamma-aminobutyric acid signaling pathway", "GABAergic synapse", "synaptic transmission", "GABAergic synaptic signaling", and "trans-synaptic signaling”. These genes were mainly involved in synaptic membrane and GABA receptor complex for cellular components (CC) and in GABA receptor activity, synaptic membrane for molecular functions (MF) (Figure 2A).
A



B



Figure 2 GO enrichment analysis and KEGG pathway analysis of 10 common genes. (A) GO analysis for ten target genes of GABRG2 and VPA-induced ADRs. Polka dots, triangles, and squares refer to biological process (BP), cellular components (CC), and molecular function (MF), respectively. (B) The KEGG pathway analysis of VPA-induced ADRs. Gene ratio represents the ratio of enriched genes to all target genes, and counts refer to the number of the enriched genes. 
Additionally, KEGG pathway and WikiPathways analysis showed that the primary pathways related to these target genes were involved in GABA receptor signaling, GABAergic synapse, taste transduction, mBDNF and proBDNF regulation of GABA neurotransmission (Figure 2B). These results reveal that GABRG2 exerts a key role in VPA-induced dADRs and weight gain likely through regulating the activities of GABA receptor complex and synaptic membrane.

\section{Discussion}

In our study, $45.6 \%$ of children with epilepsy exhibited seizure to VPA treatment and developed uncontrolled seizures, in particular younger children and those received VPA combined with other ASM. Furthermore, a high incidence of ADRs related to VPA was observed in 96 children with epilepsy. Among all types of ADRs, dADRs and weight gain were the major side effects of VPA, which were consistent with the previous evidences. ${ }^{30,31}$ The high incidence rates of dose-related ADRs in children might be due to incomplete function of liver and kidney for children, the usage of ASM polytherapy, and long-term therapy course of epilepsy.

The present study revealed a significant association of GABRG2 rs211037 polymorphism with the efficacy and ADRs related to VPA in Chinese southern children with epilepsy. Patients carrying GABRG2 rs211037 CC genotype exhibited better drug response, higher risk of VPAinduced dADRs, and fewer predispositions to weight gain to VPA compared to CT and TT genotypes. To our knowledge, our study was the first to reveal the correlation between GABRG2 rs211037 and ADRs to VPA. However, different results about association between GABRG2 rs211037 and ASM response were obtained among previous studies, likely because of differences in ethnicities of study participants, types of epilepsy, ASM for treatment, and sample size. ${ }^{20,22}$

Numerous evidences showed that physiologic, ontogenic, environmental, and genetic factors could impact the absorption, distribution, metabolism, and elimination of drugs, leading to differential sensitivity to drug exposure for children. Genetic variation is regarded as an important factor to influence drug response and ADRs induced by VPA in epileptic patients through pharmacokinetics and pharmacodynamics. ${ }^{27,32,33}$ However, the role of GABRG2 rs211037 variation in the response to VPA for children with epilepsy remains unknown.
$\mathrm{GABA}_{\mathrm{A}}$ receptors and glutamate neurotransmitters were believed as molecular targets for many ASM, based on an association between the genetic alteration of the $\mathrm{GABA}_{\mathrm{A}}$ receptors and the development of non-response to $\mathrm{ASM}^{12}$ It should be recognized that various ASM exert antiepileptic effects via GABA binding. ${ }^{34}$ Alteration of sites associated with $\mathrm{GABA}_{\mathrm{A}}$ receptors led to a poor response to ASM for epileptic patients. ${ }^{35}$ GABRG2 gene, located in position 588 of exon 5 , encodes for the $\gamma 2$ subunit which influence the action of post-synaptic transmission of $\mathrm{GABA}_{\mathrm{A}}$ receptors. ${ }^{34}$ GABRG2 was considered as a potential interactive target for $\mathrm{ASM}^{18}$ GABRG2 rs211037 was found to be associated with the splicing and transcriptional organization. Moreover, variations of $G A B R G 2$ lead to functional loss of the $\mathrm{GABA}_{\mathrm{A}}$ receptors through decreasing GABA expression or deactivation, resulting in epilepsy or recurrent epileptic seizures. ${ }^{36}$ The association of decreased drug efficacy with GABRG2 rs211037 polymorphism in our study may be explained due to overstimulated glutamate excitation, resistant genes triggered, and changed configuration and functional properties of $\mathrm{GABA}_{\mathrm{A}}$ receptors. ${ }^{20}$

The underlying mechanisms of GABRG2 in VPAinduced dADRs and weight gain were further explored by network pharmacological analysis. Ten target genes of GABRG2 related to the two above ADRs were identified and enriched in "GABAergic synapse", "taste transduction", and "GABA receptor signaling pathways". The findings from network pharmacological analysis in this study confirmed the key role of GABAergic synapse pathway in food intake and weight increased as evidenced by previous studies. $^{37}$ An abnormal activation in GABA expression related to GABAergic transmission may cause a change in appetite, and obesity. ${ }^{38-40} \mathrm{~A}$ study in mice suggested that changes in taste signaling taste signaling pathways could trigger changes in appetite, which -might lead to obesity. ${ }^{41,42}$ Evidence revealed that the sensitivity of GABA played a crucial role in determining fat depotselective adipose tissue macrophage infiltration in obesity. ${ }^{43}$ GABA receptor binding was found to modulate obesity and prioritizing a high-fat diet. ${ }^{44}$ Our data revealed that GABRG2 exerted crucial effects on VPA-induced dADRs and weight gain likely via multiple targets/pathways, and the potential mechanisms will be further elucidated in our on-going study.

Due to the unpredictability of ADRs occurrence, only a relatively small sample size was used in this study, which may result in non-significant differences on the frequency distribution of GABRG2 rs211037 for VPA-related ADRs 
from other systems. Furthermore, findings from network pharmacological analysis lacked further experimental validation including in vitro and in vivo studies. In addition, limitation of epigenetic data may limit the extent of our study. Therefore, further study with increased sample size is need to explore the role of $G A B R G 2$ rs211037 in VPA-related ADRs. $G A B R G 2$ mRNA and protein expression in different genotypes of $G A B R G 2 \mathrm{rs} 211037$ should be determined to provide insights into the molecular mechanisms of GABRG2 in drug safety for children with epilepsy.

\section{Conclusion}

In this study, gene polymorphism of GABRG2 rs211037 was correlated to drug response, dADRs and weight gain in children who received VPA. Carriers of GABRG2 rs211037 CC genotype were inclined to exhibit seizurefree to VPA and those with CT genotype conversely. GABRG2 rs211037 CC genotype tended to be a risk factor for dADRs but a protective factor for weight gain in children with VPA treatment. Ten common genes of GABRG2 in VPA-induced dADRs and weight gain were identified using network pharmacological analysis, which were involved in "GABAergic", "taste transduction" and "GABA receptor signaling" pathways. Further study will elucidate the role of GABRG2 in VPA-related efficacy and safety and provide insights into the involved molecular mechanisms.

\section{Abbreviations}

ADRs, adverse drug reactions; ASM, anti-seizure medications; BP, biological process; cADRs, cutaneous adverse drug reactions; $\mathrm{CC}$, cellular components; dADRs, digestive adverse drug reactions; GABA, gamma-amicabilityric acid; GABRG2, gamma-aminobutyric acid type A receptor subunit gamma 2; GO, Gene Ontology; KEGG, Kyoto Encyclopedia of Genes and Genomes; LEV, levetiracetam; LTG, lamotrigine; MF, molecular function; nADRs, neurogenic adverse drug reactions; OXC, oxcarbazepine; SNP, single nucleotide polymorphism; TPM, topiramate; VPA, Valproic acid.

\section{Data Sharing Statement}

The data that support the findings of this study are available from the corresponding author (Dr Xiaomei Fan) upon reasonable request.

\section{Acknowledgments}

This study was supported by the National Natural Science Foundation of China [Grant 81603185] and the Natural
Science Foundation of Guangdong province [Grant 2020A1515011452].

\section{Disclosure}

The authors declare that the research was conducted in the absence of any commercial or financial relationships that could be construed as a potential conflict of interest.

\section{References}

1. Löscher W, Potschka H, Sisodiya SM, Vezzani A. Drug resistance in epilepsy: clinical impact, potential mechanisms, and new innovative treatment options. Pharmacol Rev. 2020;72(3):606-638. doi:10.1124/ pr.120.019539

2. Fine A, Wirrell EC. Seizures in children. Ped Rev. 2020;41 (7):321-347. doi:10.1542/pir.2019-0134

3. Tan NN, Tang HL, Lin GW, et al. Epigenetic downregulation of Scn3a expression by valproate: a possible role in its anticonvulsant activity. Mol Neurobiol. 2017;54(4):2831-2842. doi:10.1007/s12035016-9871-9

4. Shinnar S, O'Dell C, Berg AT. Mortality following a first unprovoked seizure in children: a prospective study. Neurology. 2005;64 (5):880-882. doi:10.1212/01.wnl.0000152893.72146.92

5. Garcia Pierce J, Aronoff S, Del Vecchio M. Systematic review and meta-analysis of seizure recurrence after a first unprovoked seizure in 815 neurologically and developmentally normal children. $J$ Child Neurol. 2017;32(13):1035-1039. doi:10.1177/0883073817726461

6. Sartori S, Nosadini M, Tessarin G, et al. First-ever convulsive seizures in children presenting to the emergency department: risk factors for seizure recurrence and diagnosis of epilepsy. Dev Med Child Neurol. 2019;61(1):82-90. doi:10.1111/dmcn.14015

7. Jahromi SR, Togha M, Fesharaki SH, et al. Gastrointestinal adverse effects of antiepileptic drugs in intractable epileptic patients. Seizure. 2011;20(4):343-346. doi:10.1016/j.seizure.2010.12.011

8. Belcastro V, D'Egidio C, Striano P, Verrotti A. Metabolic and endocrine effects of valproic acid chronic treatment. Epilepsy Res. 2013;107(1-2):1-8. doi:10.1016/j.eplepsyres.2013.08.016

9. Zhang H, Lu P, Tang HL, et al. Valproate-induced epigenetic upregulation of hypothalamic Fto expression potentially linked with weight gain. Cell Mol Neurobiol. 2020. doi:10.1007/s10571-02000895-2

10. Zhu MM, Li HL, Shi LH, Chen XP, Luo J, Zhang ZL. The pharmacogenomics of valproic acid. J Hum Genet. 2017;62(12):1009-1014. doi:10.1038/jhg.2017.91

11. Berg AT, Langfitt JT, Testa FM, et al. Global cognitive function in children with epilepsy: a community-based study. Epilepsia. 2008;49 (4):608-614. doi:10.1111/j.1528-1167.2007.01461.x

12. Rogawski MA, Löscher W. The neurobiology of antiepileptic drugs. Nat Rev Neurosci. 2004;5(7):553-564. doi:10.1038/nrn1430

13. Cunningham MO, Woodhall GL, Jones RS. Valproate modifies spontaneous excitation and inhibition at cortical synapses in vitro. Neuropharmacology. 2003;45(7):907-917. doi:10.1016/s00283908(03)00270-3

14. Löscher W. Valproate: a reappraisal of its pharmacodynamic properties and mechanisms of action. Prog Neurobiol. 1999;58(1):31-59. doi:10.1016/s0301-0082(98)00075-6

15. Macdonald RL, Kang JQ. Molecular pathology of genetic epilepsies associated with GABAA receptor subunit mutations. Epilepsy Curr. 2009;9(1):18-23. doi:10.1111/j.1535-7511.2008.01278.x

16. Bhat MA, Guru SA, Mir R, et al. Association of GABAA receptor gene with epilepsy syndromes. $J$ Mol Neurosci. 2018;65(2):141-153. doi:10.1007/s12031-018-1081-7 
17. Haerian BS, Baum L, Kwan P, et al. Contribution of GABRG2 polymorphisms to risk of epilepsy and febrile seizure: a multicenter cohort study and meta-analysis. Mol Neurobiol. 2016;53 (8):5457-5467. doi:10.1007/s12035-015-9457-y

18. Wang S, Zhang X, Zhou L, Wu Q, Han Y. Analysis of GABRG2 C588T polymorphism in genetic epilepsy and evaluation of GABRG2 in drug treatment. Clin Transl Sci. 2021. doi:10.1111/cts.12997

19. Milanovska M, Cvetkovska E, Panov S. Association of rs211037 GABRG2 gene polymorphism with susceptibility to idiopathic generalized epilepsy. Medicinski Glasnik. 2021;18(2). doi:10.17392/1367-21

20. Abou El Ella SS, Tawfik MA, Abo El Fotoh WMM, Soliman OAM. The genetic variant "C588T" of GABARG2 is linked to childhood idiopathic generalized epilepsy and resistance to antiepileptic drugs. Seizure. 2018;60:39-43. doi:10.1016/j.seizure.2018.06.004

21. Gitaí LL, de Almeida DH, Born JP, et al. Lack of association between rs211037 of the GABRG2 gene and juvenile myoclonic epilepsy in Brazilian population. Neurol India. 2012;60(6):585-588. doi:10.4103/0028-3886.105191

22. Balan S, Sathyan S, Radha SK, Joseph V, Radhakrishnan K, Banerjee M. GABRG2, rs211037 is associated with epilepsy susceptibility, but not with antiepileptic drug resistance and febrile seizures. Pharmacogenet Genomics. 2013;23(11):605-610. doi:10.1097/ fpc. 000000000000000

23. Butilă AT, Zazgyva A, Sin AI, Szabo ER, Tilinca MC. GABRG2 C588T gene polymorphisms might be a predictive genetic marker of febrile seizures and generalized recurrent seizures: a case-control study in a Romanian pediatric population. Arch Med Sci. 2018;14 (1):157-166. doi:10.5114/aoms.2016.63739

24. Ponnala S, Chaudhari JR, Jaleel MA, et al. Role of MDR1 C3435T and GABRG2 C588T gene polymorphisms in seizure occurrence and MDR1 effect on anti-epileptic drug (phenytoin) absorption. Genet Test Mol Biomarkers. 2012;16(6):550-557. doi:10.1089/gtmb.2011.0225

25. Kumari R, Lakhan R, Kalita J, Misra UK, Mittal B. Association of alpha subunit of GABAA receptor subtype gene polymorphisms with epilepsy susceptibility and drug resistance in north Indian population. Seizure. 2010;19(4):237-241. doi:10.1016/j.seizure.2010.02.009

26. Scheffer IE, Berkovic S, Capovilla G, et al. ILAE classification of the epilepsies: position paper of the ILAE Commission for Classification and Terminology. Epilepsia. 2017;58(4):512-521. doi:10.1111/epi.13709

27. Wolking S, Moreau C, Nies AT, et al. Testing association of rare genetic variants with resistance to three common antiseizure medications. Epilepsia. 2020;61(4):657-666. doi:10.1111/epi.16467

28. Tomson T, Battino D, Perucca E. Valproic acid after five decades of use in epilepsy: time to reconsider the indications of a time-honoured drug. Lancet Neurol. 2016;15(2):210-218. doi:10.1016/s14744422(15)00314-2

29. Fan X, Chen Y, Li W, et al. Genetic polymorphism of ADORA2A is associated with the risk of epilepsy and predisposition to neurologic comorbidity in Chinese Southern Children. Front Neurosci. 2020;14:590605. doi:10.3389/fnins.2020.590605

30. Bai X, Xu C, Wen D, et al. Polymorphisms of peroxisome proliferator-activated receptor $\gamma(\operatorname{PPAR} \gamma)$ and cluster of differentiation 36 (CD36) associated with valproate-induced obesity in epileptic patients. Psychopharmacol. 2018;235(9):2665-2673. doi:10.1007/ s00213-018-4960-2

Pharmacogenomics and Personalized Medicine

\section{Publish your work in this journal}

Pharmacogenomics and Personalized Medicine is an international, peer-reviewed, open access journal characterizing the influence of genotype on pharmacology leading to the development of personalized treatment programs and individualized drug selection for improved safety, efficacy and sustainability. This journal is indexed
31. Kaushik S, Chopra D, Sharma S, Aneja S. Adverse drug reactions of anti-epileptic drugs in children with epilepsy: a cross-sectional study. Curr Drug Saf. 2019;14(3):217-224. doi:10.2174/ 1574886314666190311112710

32. Lauschke VM, Ingelman-Sundberg M. Prediction of drug response and adverse drug reactions: from twin studies to next generation sequencing. Eur J Pharm Sci. 2019;130:65-77. doi:10.1016/j. ejps.2019.01.024

33. Xu S, Chen Y, Zhao M, Guo Y, Wang Z, Zhao L. Population pharmacokinetics of valproic acid in epileptic children: effects of clinical and genetic factors. Eur J Pharm Sci. 2018;122:170-178. doi:10.1016/j.ejps.2018.06.033

34. Olsen RW. GABA(A) receptor: positive and negative allosteric modulators. Neuropharmacology. 2018;136(Pt $\quad$ A):10-22. doi:10.1016/j.neuropharm.2018.01.036

35. Salam SM, Rahman HM, Karam RA. GABRG2 gene polymorphisms in Egyptian children with simple febrile seizures. Indian J Pediatr. 2012;79(11):1514-1516. doi:10.1007/s12098-011-0564-0

36. Krampfl K, Maljevic S, Cossette P, et al. Molecular analysis of the A322D mutation in the GABA receptor alpha-subunit causing juvenile myoclonic epilepsy. Eur $J$ Neurosci. 2005;22(1):10-20. doi:10.1111/j.1460-9568.2005.04168.x

37. Fan H, Wu Y, Zhou X, et al. Pathway-based genome-wide association studies for two meat production traits in Simmental cattle. Sci Rep. 2015;5:18389. doi:10.1038/srep18389

38. Zhang X, van den Pol AN. Rapid binge-like eating and body weight gain driven by zona incerta GABA neuron activation. Science. 2017;356(6340):853-859. doi:10.1126/science.aam7100

39. Zhu C, Jiang Z, Xu Y, et al. Profound and redundant functions of arcuate neurons in obesity development. Nat Metabol. 2020;2 (8):763-774. doi:10.1038/s42255-020-0229-2

40. Sandoval-Salazar C, Ramírez-Emiliano J, Trejo-Bahena A, OviedoSolís CI, Solís-Ortiz MS. A high-fat diet decreases GABA concentration in the frontal cortex and hippocampus of rats. Biol Res. 2016;49:15. doi:10.1186/s40659-016-0075-6

41. Choo E, Wong L, Chau P, Bushnell J, Dando R. Offspring of obese mice display enhanced intake and sensitivity for palatable stimuli, with altered expression of taste signaling elements. Sci Rep. 2020;10 (1):12776. doi:10.1038/s41598-020-68216-7

42. Avau B, Bauters D, Steensels S, et al. The gustatory signaling pathway and bitter taste receptors affect the development of obesity and adipocyte metabolism in mice. PLoS One. 2015;10(12):e0145538. doi: 10.1371 journal.pone. 0145538

43. Hwang I, Jo K, Shin KC, et al. GABA-stimulated adipose-derived stem cells suppress subcutaneous adipose inflammation in obesity. Proc Natl Acad Sci U S A. 2019;116(24):11936-11945. doi:10.1073/ pnas. 1822067116

44. Covelo IR, Patel ZI, Luviano JA, Stratford TR, Wirtshafter D. Manipulation of GABA in the ventral pallidum, but not the nucleus accumbens, induces intense, preferential, fat consumption in rats. Behav Brain Res. 2014;270:316-325. doi:10.1016/j.bbr.2014.05.032

on the American Chemical Society's Chemical Abstracts Service (CAS). The manuscript management system is completely online and includes a very quick and fair peer-review system, which is all easy to use. Visit http://www.dovepress.com/testimonials.php to read real quotes from published authors. 